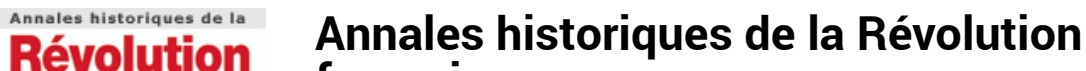

française française

341 | juillet-septembre 2005

Varia

\section{La peur de la Révolution française à Malte}

\section{Frans Ciappara}

\section{OpenEdition}

Journals

Édition électronique

URL : https://journals.openedition.org/ahrf/1614

DOI : 10.4000/ahrf.1614

ISSN : 1952-403X

Éditeur :

Armand Colin, Société des études robespierristes

Édition imprimée

Date de publication : 1 septembre 2005

Pagination : 53-68

ISSN : 0003-4436

\section{Référence électronique}

Frans Ciappara, «La peur de la Révolution française à Malte », Annales historiques de la Révolution française [En ligne], 341 | juillet-septembre 2005, mis en ligne le 15 septembre 2008, consulté le 24 avril 2022. URL : http://journals.openedition.org/ahrf/1614; DOI : https://doi.org/10.4000/ahrf.1614

Ce document a été généré automatiquement le 24 avril 2022.

Tous droits réservés 


\section{La peur de la Révolution française à Malte}

\section{Frans Ciappara}

1 Malte ne fut pas saisie par la "grande peur » de la Révolution française ${ }^{1}$. Le peuple ne s'arma pas dans un réflexe d'autodéfense et aucune révolte agraire ne fut enregistrée ; pas plus qu'aucun château ne fut réduit en flammes. Mais la peur était tout de même dans l'air, la peur d'une rébellion ou d'une invasion; comme le décrivent parfaitement bien un ensemble de dépêches trouvées aux Archives nationales de Naples.

2 Le 11 août 1789, l'Assemblée nationale française fit trembler l'Ordre des chevaliers de Saint-Jean jusque dans ses fondations lorsqu'elle abolit la dîme ${ }^{2}$. La dîme formait la plus grosse part des revenus des commanderies ou des domaines en France. Dans ces circonstances désastreuses, le 25 septembre, le Grand Maître Emmanuel Rohan fit comprendre au roi de Naples que les services de l'Ordre s'étendaient à toute la chrétienté, assurant refuge et protection aux navigateurs, et lui demanda assistance [50 ${ }^{3}$. Ferdinand réalisa l'utilité que son royaume tirait de son commerce avec Malte et ordonna à son ministre Pignatelli d'observer les événements sur l'île avec vigilance ${ }^{4}$.

3 L'existence même de l'Ordre était menacée et M. Camus réclamait son abolition avec beaucoup d'enthousiasme et de zèle ${ }^{5}$. Mais les chambres de commerce de Marseille et de Bordeaux démontrèrent toutes deux les avantages que la France tirait de Malte ${ }^{6}$. Mayer fit remarquer la même chose à l'Assemblée nationale, tout en affirmant que l'Ordre dépendait du pape uniquement pour les questions d'ordre spirituel ${ }^{7}$.

4 Le bailli de Virieu, chargé d'affaires de l'Ordre à Paris, était désespéré. Afin de se faire bien voir des révolutionnaires, les «Langues" françaises contribuèrent au «don patriotique » et offrirent un quart de leurs revenus ${ }^{8}$. Rohan envoya des lettres à Louis XVI, ainsi qu'à tous les rois catholiques d'Europe, et surtout au pape, demandant leur médiation 9. Il donna également l'ordre d'interdire aux chevaliers de prendre la moindre part dans la contre-révolution ${ }^{10}$. Il n'autorisa pas non plus la trésorerie de l'Ordre à prêter 200000 scudi au marquis de la Lare. Ce chevalier de l'Ordre de SaintLouis originaire d'Aix-en-Provence était un ennemi juré de la Révolution et partisan du 
comte d'Artois, le frère cadet du roi. Il était également un grand antagoniste de Mirabeau, ce qui lui avait valu de courir le risque d'être lynché par le peuple ${ }^{11}$.

Rohan était conciliant et abolit la propagande anti-révolutionnaire. Le dernier jour du carnaval de 1790, deux chevaliers italiens masqués firent la satire de l'Assemblée nationale et de la Nation française. Le chevalier Siennois Sansedoni était déguisé en démon avec une longue queue et une pancarte accrochée aux épaules disant «le démon de la liberté ». Il montait un âne couronné de nombreux rubans blancs, rouges et bleus, lui-même affublé d'une affiche disant : «Je ne suis pas un âne mais une bête sauvage. Le démon de la liberté a fait de moi son esclave ». Un autre chevalier italien, le Florentin Bonsignori, qui portait une chemise déchirée, tenait la queue du démon et lui disait, « Mon ami, donne moi ma robe », à quoi le démon répondait, « Tu n'en n'as jamais eu ». Les deux Hospitaliers furent arrêtés ${ }^{12}$.

6 Cependant, la situation à l'étranger était bien plus grave que les intérêts de la petite île de Malte. Avec la tentative d'évasion de la famille royale, à Varennes, l'ordre, dont les revenus avaient subventionné l'entreprise, perdit le dernier sympathisant sur lequel il pouvait encore compter. De même, avec l'invasion de la France par des forces ennemies, les chances de survie de l'Ordre furent anéanties. Le coup fatal s'abattit le 19 septembre 1792, lorsque les terres de l'Ordre furent confisquées. Le bailli de Foresta, réceptionnaire de l'Ordre à Marseille, fut envoyé à Paris pour protester contre ce décret ${ }^{13}$. Il revint les mains vides, mais le Grand Maître était encore conciliant. Le 22 février 1793, quelques quarante Hospitaliers français se réunirent au matin sur la place principale de La Valette. Leur intention était de se rendre chez M. Comeau, l'ancien ministre du roi de France, afin d'enlever le blason royal en haut de la porte de sa résidence. Selon eux, dès lors que le monarque était mort et qu'une constitution républicaine était en place, le ministre ne pouvait plus exposer les insignes royaux. Le préfet de police les persuada de rentrer chez eux ${ }^{14}$, mais le Grand Maître, afin de se mettre en conformité avec les réclamations de la Convention, fit enlever le blason contesté ${ }^{15}$. Rohan fit aussi interdire que soient vendues au public ${ }^{16}$ des images représentant « l'exécution barbare de l'infortuné Louis XVI » - même si lui-même et son conseil assistèrent à une messe à la mémoire de Marie-Antoinette ${ }^{17}$.

7 Sur une chose cependant l'Ordre tint bon. Le 7 mars 1793, le Comité de Salut public de la Convention nationale conçut un plan pour forcer Malte à abandonner sa «neutralité stérile » et à participer au commerce de la France. Au moyen de ce plan, approuvé par la Commission des Subsistances et Approvisionnements, l'île allait devenir un dépôt pour les céréales françaises en échange d'une promesse de défense ${ }^{18}$. Les Maltais devaient être payés en moins de huit jours par un agent français, mais ils pouvaient également être payés en nature, par exemple, en beaux tissus, produits coloniaux, porcelaine, verre, pierres précieuses et autres marchandises. Accompagné d'un marchand et de deux Français révolutionnaires ${ }^{19}$, Foresta arriva à Gênes ${ }^{20}$. Le Grand Maître rejeta ce plan et donna des ordres pour que les trois représentants de la commission - une guillotine ayant été prétendument trouvée parmi leurs biens - ne fussent pas autorisés à débarquer à Malte ${ }^{21}$.

8 L'Ordre ne devenait pas l'allié de la France, mais il n'aidait pas non plus ouvertement ses adversaires ${ }^{22}$. En 1795 le chevalier Ferret arriva à Malte pour lever un régiment de cavalerie pour l'armée du prince de Condé. Rohan et la congrégation d'État désapprouvèrent, et ceux qui s'engagèrent ne furent pas autorisés à quitter l'île. Ainsi, un certain Reno, un Maltais, fut menacé de mort s'il continuait à engager des hommes ; 
et il fut interdit aux bateaux du port de prendre à bord plus de deux Maltais ${ }^{23}$. Le Grand Maître montra également sa bonne volonté l'année suivante lorsqu'une frégate française fut poussée vers le port de Marsamxett par des vents contraires. Contre l'avis de quelques chevaliers, Rohan donna au capitaine toute l'aide nécessaire pour continuer son chemin vers Marseille ${ }^{24}$.

9 Mais le temps qui passait rendait la cause de l'Ordre désespérée. Le 13 juillet 1796 le Conseil nomma Fra Giuseppe d'Hannovile ambassadeur extraordinaire afin de négocier avec les Français la restitution de ses biens par l'entremise du roi d'Espagne ${ }^{25}$. Les négociations échouèrent, sous le prétexte que le Directoire devait d'abord faire la paix avec d'autres pouvoirs avant de parvenir au moindre accord avec l'Ordre ${ }^{26}$.

10 Ce ne fut cependant pas la seule perte de ses propriétés terriennes ${ }^{27}$ qui appauvrit l'Ordre ${ }^{28}$. Dans ces circonstances désastreuses, de nombreux chevaliers revinrent à Malte, ce qui augmenta les problèmes de trésorerie de l'Ordre. Parmi ces émigrés se trouvait Philippe Marie de Andlau qui fit appel au pape pour l'aider dans son extrême misère. Il était assuré de trouver à Malte une "table et une maison" ${ }^{29}$. Afin de renflouer les caisses, l'impôt sur le vin fut augmenté en $1793{ }^{30}$, mais l'Ordre avait besoin de fonds plus tangibles. À cette fin, le Conseil désigna, le mardi 3 novembre 1795, quatre "grandes Croix " ${ }^{31}$ qui, avec les procurateurs et le secrétaire des finances, devaient proposer des solutions pour améliorer la situation financière. Une de ces mesures fut que l'affranchissement postal devait désormais être payé par tous ${ }^{32}$, à l'exception de l'inquisiteur ${ }^{33}$. L'Ordre essayait d'économiser autant qu'il le pouvait. Au 1er septembre 1796, il avait réussi à diminuer ses dépenses annuelles de 240000 scudi maltais, mais il manquait encore 160000 scudi. Il fut alors décidé d'augmenter les responsions des commanderies de toutes les Langues ${ }^{34}$.

11 Lorsque la Révolution éclata, le gouvernement prit les mesures militaires nécessaires pour défendre l'île, surtout lorsque la rumeur circula, en novembre 1792, que La Touche approchait de Malte avec sa flotte. Le bailli du Tillet, qui avait servi en France, ainsi que le chevalier commandeur Thurn furent les deux généraux nommés pour assurer la défense de l'île. Le « corps de chasseurs » fut doublé et passa de 500 à 1000 hommes ; ses membres firent de nombreuses manœuvres et furent placés le long de la côte. Les soldats des galères et des vaisseaux furent envoyés protéger les forts de StAnge et Ricasoli, tandis que les régiments « de la campagne » et les milices des villes furent mis en alerte. Le vaisseau St-Zacharie et la frégate Ste-Élisabeth surveillaient les baies et ne laissaient personne accoster qui n'ait auparavant reçu la «libre pratique ». Ces mesures permirent d'empêcher ces espions impies d'entrer furtivement dans le pays pour séduire et conspirer contre l'ordre public. Le chantier naval, le dépôt de poudre et autres lieux stratégiques furent également placés sous haute surveillance ${ }^{35}$, de même que les portes principales de la capitale ${ }^{36}$.

12 Mais bien plus que cela encore, des mesures furent prises contre « les individus obsédés par les étourdissements qui régnaient alors en France et dans les pays voisins " ${ }^{37}$. Le président de la Cour supérieure de justice, Grimaldi, fut nommé à la tête de la congrégation criminelle d'État afin d'engager des poursuites contre ceux qui étaient "entachés de maximes françaises" ${ }^{38}$. Il était aidé du procureur général et du commissaire de police ${ }^{39}$ tandis que Rohan se confiait à lui et lui montrait la correspondance secrète venant de Paris ${ }^{40}$.

13 On s'occupa de " ceux qui défiaient la paix » d'une manière expéditive ${ }^{41}$ et ils furent exilés ${ }^{42}$. Cette mesure s'appliqua plus particulièrement aux Français, même s'ils 
résidaient sur l'île depuis longtemps et exerçaient un métier ou dirigeaient un commerce ${ }^{43}$. Trois sergents français du "régiment de Malte» furent également déportés le 17 août 1794 pour avoir tenu entre eux des réunions secrètes. Il ne restait plus aucun Français, deux exceptés, qui avaient servi dans la contre-révolution et étaient réputés dignes de confiance ${ }^{44}$. Guillaume Laurier arriva à Malte en juillet 1794 sur un vaisseau vénitien. Il avait été expulsé du royaume des Deux Siciles sur la présomption d'être parmi ceux qui avaient provoqué des troubles dans ce royaume. On ne lui permit même pas de débarquer ${ }^{45}$.

Ce n'était pas seulement les Français qui étaient bannis, mais aussi tous les étrangers suspectés d'adhérer aux maximes révolutionnaires. Carolina Giardinelli, « une perfide jacobine » ${ }^{46}$, raconta à quelques chevaliers français que « les Napolitains n'étaient plus tenus d'obéir à l'autorité de leur roi, parce qu'elle était abusive " ${ }^{47}$. Un marchand sicilien, du nom de Zappalà, eut même l'audace de dire à un homme dont il croyait qu'il nourrissait les mêmes sentiments révolutionnaires que lui :

«L'Europe est dans le désarroi, et aussi longtemps qu'il restera un seul roi, elle ne pourra pas être en paix parce qu'il est difficile et nuisible de se soumettre à une seule personne. » ${ }^{48}$

L'expulsion fut aussi le sort réservé à une dame allemande, Elisabeth Dalmazzo, qui portait un serre-tête aux couleurs françaises ${ }^{49}$.

La peur saisit la Nation. Selon Elizabeth Schermerhorn, qui donne une description vivante et détaillée de ces présomptions et rumeurs insidieuses, tout étranger était un espion, et les magasins et les maisons soupçonnés de cacher des armes ou des documents secrets étaient fouillés.

«Les auberges, où jadis les sujets les plus incendiaires n'étaient que butins, suprématie, inquisiteurs et élections, retentissaient maintenant de débats véhéments sur les classes privilégiées et les droits de l'homme; et le vieil apoplectiques Baillis dissertait avec colère à propos de la théorisation grotesque de la jeunesse, et sur l'audace des jeunes, qui n'avaient pas encore mérité leur « Croix » et leurs Commanderies, et prétendaient prouver que l'Ordre militaire souverain était incompatible avec le progrès de l'humanité. Lorsqu'une douzaine de chevaliers français, voire plus, quittèrent l'ordre, le bruit couru qu'ils étaient partis rejoindre les révolutionnaires. » ${ }^{50}$

En 1791, quelques Hospitaliers français en vinrent aux mains avec un groupe de marins italiens. On commença bientôt à dire que la principale préoccupation des chevaliers était de piller le Trésor public, les maisons des Maltais les plus riches et de quitter l'île. D'autres croyaient, conjointement avec de nombreux habitants mécontents, que les Français avaient l'intention de fomenter une révolte.

Le même doute survint plus tard cette année-là, lorsqu'en juin, Naples envoya deux navires de guerre pour surveiller le centre de la Méditerranée. Cet événement, s'associant à un ordre du gouvernement maltais de fortifier les forts par des canons, donna aux habitants des raisons de croire que l'île n'était pas complètement sûre. Peutêtre qu'une armée allait l'attaquer, peut-être qu'une révolte locale allait éclater ${ }^{51}$. La peur augmenta en juillet lorsqu'une liste d'appelés fut dressée. Une invasion des Anglais ou des Turcs était imminente; d'autres imaginèrent des «événements plus meurtriers ${ }^{52}$.

19 En 1794, on rapporta que des chants révolutionnaires français traduits en maltais étaient chantés dans un des villages. Les gens furent arrêtés, mais une enquête et un contre-interrogatoire sérieux prouvèrent qu'il ne s'agissait que d'une fausse rumeur ${ }^{53}$. 
Un peu plus tard, un paysan de Zebbug accusa secrètement de sédition une bonne famille du même village. Mais, après une enquête zélée, il fut prouvé que les accusations étaient fausses, et le délateur confessa sa calomnie. Il fut exilé à Gozo ; et bien que ses protecteurs eussent essayé de le faire envoyer dans d'autres villages, le Grand Maître resta ferme dans sa décision d'éloigner de Malte cette "graine dévastatrice de la calomnie $»^{54}$.

Selon Grimaldi, les Maltais profitèrent joyeusement de l'occasion de prouver leur immuable loyauté envers la religion ${ }^{55}$. Toujours reconnaissants envers "Son Éminence » et déterminés à être fidèles envers leur gouvernement, ils offrirent de grosses sommes d'argent pour la défense de l'État ${ }^{56}$. Mais les traîtres ne manquaient pas.

21 Lorsque Grimaldi apprit, par conséquent, la «conduite scandaleuse» de deux dominicains, Fra Alessandro Grech et Fra Tommaso Vassallo, tous deux résidents au couvent de Rabat, il exhorta le Grand Maître à les envoyer en Sicile pour les punir. Ces disciples du père Levante avaient essayé de répandre leurs «maximes impies » parmi leurs compatriotes. Ils disaient sans cesse du mal du gouvernement de l'Ordre et prédisaient souvent la révolte de la Nation maltaise, sous le prétexte d'être très contrariés et accablés par le manque de denrées alimentaires et de libre-échange. Ce serait au grand soulagement et à l'avantage des Maltais, affirmaient les deux religieux, si un autre pays intervenait en leur faveur ${ }^{57}$.

Une lettre datée du 19 mai 1792 informa le gouvernement qu'une conspiration se tramait à Malte. Elle était financée par les riches marchands de Burmola et de Zejtun qui continuaient à commercer avec Barcelone. Ce fut le notable «Charles Zammit » ${ }^{58}$ qui divulgua le complot à Virieu ; complot qui lui avait été prétendument révélé par un certain Buhagiar, un marchand maltais de Burmula. Les instigateurs présumés étaient Samuel Caruana, ancien procureur général démis de ses fonctions pour corruption, et un certain Dr Gatt, homme de loi. Durant cette année-là, les deux hommes avaient correspondu avec Basire, vice-président du Comité de surveillance à Paris, tous les vingt jours. Dans un de ces courriers, daté du 25 mai 1792, ils se vantèrent que bientôt ils se reposeraient «à l'ombre de l'arbre de la liberté ». Les droits de l'homme allaient instaurer l'égalité tout en détruisant l'Ordre, son Grand Maître et son Conseil, pour le plus grand bien du peuple maltais qui devait endurer esclavage, tyrannie et pauvreté de leur propre gouvernement. Le chant révolutionnaire "Ça ira » avait été chanté sous les bastions du Fort St-Elme. Le nombre des disciples de cette révolution augmentait chaque jour et environ 250 personnes suivaient déjà le mouvement. Parmi eux, Paul Manduca, qui vivait dans la maison de la Baronne Vincenza, Fournier et toute sa famille, et les deux représentants de l'Inquisition, Georges Portelli et Jean Gatt. Parmi les conspirateurs présumés se trouvaient aussi Georges Olivier, neveu de l'assesseur de l'évêque Pellerano, un certain Mallia, imprimeur et chanoine de Birchircara, Emanuele Carbone, Jean-Marie Vella de la paroisse de St-Georges à Gozo, Paul Vella, secrétaire des évêques Pellerano et Labini ainsi que Jean-Marie Deguara, capitaine du tribunal ecclésiastique.

Les habitants de Zebbug devaient plus particulièrement être placés sous surveillance, parce qu'ils adhéraient presque tous à la Révolution; un d'entre eux, un certain Dimech, avait également établi une correspondance avec Basire. Les Mannarinos avaient depuis tous temps été ennemis de l'État. Dans la maison de Bartolomeo, frère du «tristement célèbre » Don Gaetane, on trouva des documents «qui faisaient 
scandaleusement référence à la folie des Français ». Il avait envoyé ses trois fils servir sur des navires français à Toulon, sous la direction du père Dimech (Testaferrata), aumônier à bord du Tonnant ${ }^{59}$. Un autre disciple de la Révolution, Grognet, qui portait la cocarde française sur son chapeau, s'enfuit à Corfou ${ }^{60}$ à bord de deux frégates françaises, avec d'autres Maltais ${ }^{61}$.

Il semble qu'une vingtaine de chevaliers aient été impliqués. Dans cette optique, le village de Birkirkara devait être placé sous surveillance, car de nombreux Hospitaliers y avaient leur résidence. Les chevaliers étaient divisés en deux camps. Les aristocrates soutenaient énergiquement l'Ordre, affirmant que ce dernier était des plus nécessaires à la France, plus particulièrement pour son commerce. Le bailli de Loras, membre influent de ce parti aristocratique, se démenait chaque jour pour augmenter le nombre de ses disciples. De l'autre côté, les démocrates, aimant la liberté par-dessus tout, ne pouvaient pas comprendre que l'égalité entre les classes sociales soit incompatible avec l'existence de la religion.

Parmi ces derniers se trouvait le bailli de Resséquer, l'ami de l'abbé de Beausset, comte de St-Victor. Il ne désirait rien d'autre que de passer quelques jours à Marseille afin de constater par lui-même les triomphes de ses habitants. St-Priest, autre partisan implacable de l'Assemblée nationale, était la personne «la plus malhonnête, vénal, discrédité, et intime confident de Dolomieu " ${ }^{62}$. Latour-Maubourg avait servi comme aide de camp du Marquis de La Fayette. Son frère aîné avait été un des trois représentants envoyés par l'Assemblée nationale pour ramener Louis XVI de Varennes en juin $1791{ }^{63}$. Par conséquent, aussitôt que ce membre d'une famille hostile à son roi arriva à Malte en provenance de Livourne en août 1794, les chevaliers des trois Langues françaises non seulement demandèrent qu'il ne fît plus partie de l'Ordre mais il présentèrent également une pétition au Grand Maître pour qu'il fût expulsé de Malte immédiatement ${ }^{64}$. Rohan ordonna à son oncle, le bailli de Belmont, chez qui Maubourg s'était installé à Floriana, de le renvoyer ${ }^{65}$. Ce sympathisant d'une "nation aveugle et stupide ${ }^{66}$ quitta Malte le 17 août $1794{ }^{67}$.

Lorsque Scaruffi, chevalier novice de Modène ${ }^{68}$, était à Catane en 1793 il eut la témérité de dire du mal de la royauté ${ }^{69}$. Le vice-roi de Sicile rapporta les faits à son souverain et lorsque Scaruffi arriva à Naples, il fut immédiatement expulsé et vint à Malte. Il quitta l'île le 23 avril 1794 après que le capitaine, qui avait d'abord refusé de le prendre à bord, eut reçu des ordres du Grand Maître. Toutefois, les chevaliers à bord refusèrent d'avoir le moindre contact avec lui pendant le voyage ${ }^{70}$.

Grimaldi ne crut pas les révélations de Zammit, affirmant qu'elles étaient fausses et improbables. Sur l'ordre du Grand Maître, il entreprit une enquête secrète et zélée et découvrit que les personnes mentionnées dans les lettres étaient soit mortes, soit complètement insoupçonnables, étant soit mineures soit très âgées. En inventant cette histoire, Zammit avait peut-être voulu obtenir des avantages personnels, ou bien avait eu l'intention de nuire au gouvernement ${ }^{71}$. Toutes les personnes qu'il cita furent tout de même placées sous haute surveillance ${ }^{72}$.

L'inquisiteur Scotti ne fut pas non plus impressionné par ces " commérages injustes ». Il croyait fermement qu'aucun motif suffisant ne se cachait derrière cette «histoire inventée de toutes pièces ", qu'il considérait fausse et sans fondement. Son successeur, Mgr Giulio Carpegna pensait la même chose, bien qu'il affirme :

«Qu'ils soient maltais ou Hospitaliers, ils ne manquent pas ici, ceux qui regardent

d'un œil favorable les Français et leurs détestables maximes. » 
C'est ce qu'il écrivit au cardinal Secrétaire d'État le 30 mars $1797^{73}$. Ce ne fut cependant qu'en juin qu'une révolte fut découverte ${ }^{74}$, et un an plus tard Napoléon balaya ces maitres tant haïs des Maltais et leur " gouvernement despotique » ${ }^{75}$.

\section{NOTES}

1.Pour ce concept voir Georges LefeBvRE, The Great Fear of 1789. Rural Panic in

Revolutionary France, traduction Joan Wнiте, Londres, 1973.

2.J.M. Thомеson, The French Revolution, Oxford, 1966, p. 160.

3.Archivio di Stato, Napoli (ASN), Affari Esteri, fasc. 739 : Rohan à Ferdinand, 25 septembre 1789.

4.ASN, Affari Esteri, fasc. 729 : Ferdinand à Pignatelli, 28 décembre 1789.

5.Elizabeth SCHERMERHORN, Malta of the Knights, Surrey, 1929, p. 292.

6.Archives de l'Inquisition, Malte (AIM), Correspondance (Corr.) 101, f. 276r : Scotti à de Zelada, 9 janvier 1790.

7.Se référer à ses Considérations politiques et commerciales sur la nécessité de maintenir l'Ordre de Malte tel qu'il est (1790). Suite aux Considérations politiques et commerciales sur la nécessité de maintenir l'Ordre de Malte tel qu'il est. Second Mémoire (1790). Réponse à la Motion de M. Camus (1790). Les intérêts de la France liés à l'existence de l'Ordre de Malte (s.d.).

Considérations politiques et commerciales sur la nécessité de maintenir l'Ordre souverain de Malte (1797).

8.AIM, Corr. 101, ff. 273v-74r : Scotti à de Zelada, 28 novembre 1789. Fortunato Panzavecchia, L'Ultimo Periodo della Storia di Malta, Malte, 1835, pp. 302-309. Roderick CAVAliero, The Last of the Crusaders, Londres, 1960, pp. 181-204.

9.AIM, Corr. 101, f. 280v : Scotti à de Zelada, 3 avril 1790.

10.Ibid., f. 322r : Scotti à de Zelada, 10 septembre 1791.

11.Ibid., f. 287r : Scotti à de Zelada, 24 juillet 1790.

12.ASN, Affari Esteri, fasc. 729 : Pignatelli à Rohan, 20 février 1790. AIM, Corr. 101, ff. 278r-79r : Scotti à de Zelada, 6 mars 1790.

13.ASN, Affari Esteri, fasc. 6832 : Rohan à Francone, 7 février. 1793. AIM, Corr. 102, ff. 142v-43r : Carpegna à de Zelada, 23 août 1795.

14.Ibid., ff. 61r-v : Carpegna à de Zelada, 23 février 1793.

15.Ibid., ff. 150v-51r : Carpegna à de Zelada, 15 décembre 1795.

16.ASN, Affari Esteri, fasc. 6833 : Grimaldi à Acton, 15 mai 1794.

17.Ibid., Grimaldi à Acton, 31 janvier 1794.

18.Ibid., Grimaldi à Acton, 10 juillet 1794. Ce plan est également cité par Pierre-JeanLouis-Ovide Doublet, Mémoires historiques sur l'invasion et l'occupation de Malte par une armée française, Paris, 1883, pp. 129-30, et par Roderick CAVALIERO, The Last of the Crusaders, Londres, 1960, p. 202.

19.ASN, Affari Esteri, fasc. 6833 : Grimaldi à Acton, 3 juillet 1794. AIM, Corr. 102, f. 114r : Carpegna à de Zelada, 26 juin 1794.

20.ASN, Affari Esteri, fasc. 6833 : Grimaldi à Acton, 14 avril 1794. 
21.Ibid., Grimaldi à Acton, 15 mai 1794.

22.Pour les accusations portées par la suite par Napoléon concernant l'aide apportée par l'Ordre aux ennemis de la France se référer à William HARDMAN, A History of Malta during the Period of the French and British Occupations, 1798-1815, Londres, 1909, pp. 65-66. 23.AIM, Corr. 102, ff. 144r-45r : Carpegna à de Zelada, 24 septembre 1795.

24.Ibid., ff. 154r-v : Carpegna à de Zelada, 20 février 1796.

25.Ibid., ff. 163v-64r : Carpegna à Busca, 14 juillet 1796. Ibid., ff. 166r-v : Carpegna à Busca, 1er septembre 1796.

26.Ibid., ff. 170v-71v : Carpegna à Busca, 24 novembre 1796.

27.Ibid., f. 61v : Carpegna à de Zelada, 7 mars 1793.

28.Ibid., f. 144v : Carpegna à de Zelada, 24 septembre 1795.

29.Ibid., f. 116r : Carpegna à de Zelada, 17 juillet 1794.

30.Ibid., f. 94v : Carpegna à de Zelada, 10 octobre 1793.

31.C'était le plus haut grade de l'Ordre.

32.AIM, Corr. 102, ff. 149r-v : Carpegna à de Zelada, 12 novembre 1795.

33.Ibid., f. 150r : Carpegna à de Zelada, 28 novembre 1795. AIM, Corr. 82, ff. 176r-v : de Zelada à Carpegna, 15 décembre 1795.

34.AIM, Corr. 102, f. 166v : Carpegna à Busca, 1 septembre 1796. Les responsions étaient un pourcentage des revenus d'une commanderie qu'un chevalier commandeur envoyait à la Trésorerie à Malte.

35.ASN, Affari Esteri, fasc. 6833 : Grimaldi à Acton, 22 mai 1794.

36.Ibid., Grimaldi à Acton, 11 septembre 1794.

37.ASN, Affari Esteri, fasc. 729 : Pignatelli à Rohan, 28 décembre 1789.

38.ASN, Affari Esteri, fasc. 6833 : Grimaldi à Acton, 2 octobre 1794.

39.Ibid., Grimaldi à Acton, 10 octobre 1794.

40.Ibid., Grimaldi à Acton, 16 juillet 1794 .

41.ASN, Affari Esteri, fasc. 6829 : Rohan à Pignatelli, 5 août 1792.

42.Ibid., Rohan à Pignatelli, 11 octobre 1792.

43.ASN, Affari Esteri, fasc. 6833 : Grimaldi à Acton, 22 mai 1794, 24 juillet 1794.

44.Ibid., Grimaldi à Acton, 21 août 1794.

45.AIM, Corr. 102, f. 116r : Carpegna à de Zelada, 16 juillet 1794.

46.ASN, Affari Esteri, fasc. 6833 : Grimaldi à Acton, 16 juillet 1794.

47.Ibid., Grimaldi à Acton, 3 juillet 1794 .

48.Ibid., Grimaldi à Acton, 16 juillet 1794 .

49.ASN, Affari Esteri, fasc. 6829 : Rohan à Pignatelli, 13 septembre 1792.

50.Elizabeth SCHERMERHORN, op. cit., pp. 297-98.

51.AIM, Corr. 101, f. 316r : Scotti à de Zelada, 4 juin 1791.

52.Ibid., f. 319r : Scotti à de Zelada, 30 juillet 1791.

53.ASN, Affari Esteri, fasc. 6833 : Grimaldi à Acton, 4 septembre 1794.

54.Ibid., Grimaldi à Acton, 25 septembre 1794.

55.ASN, Affari Esteri, fasc. 6829 : Rohan à Pignatelli, 15 novembre 1792.

56.Ibid., Rohan à Pignatelli, 22 novembre 1792.

57.ASN, Affari Esteri, fasc. 6833 : Grimaldi à Acton, 14 août 1794.

58.Voir Alain BLONDY, «Vassalli et les réfugiés Maltais, principaux agents de la culture du coton en France, 1807-1814 », Journal of Maltese Studies, nos. 23-24 (1993), pp. 202-204. 59.ASN, Affari Esteri, fasc. 6829 : Rohan à Pignatelli, 15 novembre 1792. ASN, Affari Esteri, fasc. 6832 : Grimaldi à Acton, 23 décembre 1792. 
60.AIM, Corr. 102, f. 206r : Carpegna à Busca, 10 février 1798.

61.Ibid., ff. 203r-v : Carpegna à Busca, 30 décembre 1797. À Marseille, Gaetano Darmanin et un autre soldat inconnu faisaient partie de la garde nationale et étaient tous deux amis de Jourdan, qui avait détruit Arles. Jean-Baptiste Serofel, qui vendait du coton dans les rues de Marseille et Almio, un marchand d'oranges, étaient également soupçonnés d'être impliqués dans la conspiration. ASN, Affari Esteri, fasc. 6829 : Grimaldi à Acton, 12 juillet 1792.

62.Ibid., Grimaldi à Acton, 6 avril 1792.

63.J. M. ThOMpson, op. cit., pp. 210-2, 431.

64.AIM, Corr. 102, ff. 120v-21r : Carpegna à de Zelada, 14 août 1794. ASN, Affari Esteri, fasc. 6833 : Grimaldi à Acton, 7 août 1794.

65.Ibid., Grimaldi à Acton, 14 août 1794.

66.Ibid., Grimaldi à Acton, 25 septembre 1794.

67.Ibid., Grimaldi à Acton, 21 août 1794.

68.Ibid., Grimaldi à Acton, 15 février 1794.

69.AIM, Corr. 102, f. 111r : Scotti à de Zelada, 24 avril 1794.

70.Ibid., ff. 127v-28v : Scotti à de Zelada, 18 décembre 1794.

71.ASN, Affari Esteri, fasc. 6829 : Rohan à Pignatelli, 5 août 1792.

72." ... Les intrigues des scélérats qui ont pu facilement séduire les principaux auteurs des nos anciens troubles à Malte, où les malveillants, comme il existe dans tous les États, pour se ménager par leur moyen une retraite sûre dans notre île, où ils puissent se mettre à couvert du désordre qu'ils ont mis dans le royaume et dont ils ne tarderont pas d'être les victimes », ibid., Secrétairerie de France, Paris, 23 juillet 1792.

73.AIM, Corr. 102, f. 178r : Carpegna à Busca, 30 mars 1797.

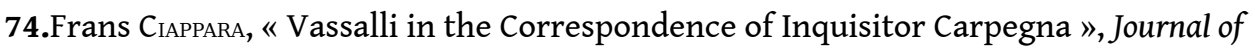
Maltese Studies, nos. 23-24 (1993), pp. 47-48.

75. « Remonstrance by the Maltese Deputies to Lord Hobart, 1802 », in Henry FRENDO (ed.), Maltese Political Development, 1798-1964. A Documentary History, Malte, 1993, p. 42. Alain Blondy, L'Ordre de Malte au XVIIIe siècle. Des dernières splendeurs à la ruine, Paris, 2002, pp. 383-449.

\section{RÉSUMÉS}

Durant la période 1789-1798 la peur à Malte était dans l'air, la peur d'une rébellion ou d'une invasion. Le gouvernement prit les mesures militaires nécessaires pour défendre l'île. Grimaldi, le président de la Cour supérieure de justice, fut nommé à la tête d'une congrégation criminelle d'État. Les partisans de la Révolution, chevaliers, maltais et étrangers furent exilés. La peur saisit la nation, spécialement quand une lettre datée du 19 mai 1792 informa le gouvernement qu'une conspiration se tramait à Malte. En juin 1797 une révolte fut découverte et un an plus tard Napoléon occupa l'île.

The fear of the French Revolution in Malta. From 1789 to 1798, the fear of a rebellion or an invasion was brewing in Malta. The government took the military measures which were 
necessary to defend the isle. Grimaldi, who was President of the higher Court of Justice, was promoted at the head of a state criminal congregation: those in favour of the revolution, such as Maltese Chevaliers or foreigners, were banished. The whole nation was panic-stricken, especially when a letter dated on the 19th of May 1792 informed the government that a conspiracy was hatching in Malta. In June 1797, a revolt was discovered. Then, one year after, Napoleon invaded the isle.

INDEX

Mots-clés : Contre-Révolution, Peur, dîme, commanderies, conspiration

\section{AUTEUR}

\section{FRANS CIAPPARA}

Université de Malte, 'Nahla', Anthony Valletta Street, B'Kara, Malta. BKR 13 